feasibility, organizational fit, and other factors unique to community-based partnership projects. RESULTS/ANTICIPATED RESULTS: We received an enthusiastic response to our RFA, based upon a long-standing program of a sister CTSA hub. Proposals received include target populations representative of our most vulnerable" children, the elderly, those lacking access to health care, and those for whom language is a barrier. One addresses the Institution's and the CEC's need to conduct community needs assessments to enable the implementation of evidence-based programs driven by data and metrics identified and developed by our communities. Each awarded proposal demonstrates a significant and sometimes critical need for the project. Partnerships are anticipated to have significant impacts on the community and its population. DISCUSSION/SIGNIFICANCE OF FINDINGS: We generate, test, and disseminate team science, education and best practices through stakeholder involvement. Our Community Health Small Grants program aims to involve community in our scientific teams and to involve academics in community-derived projects as well as foster relationships and trust.

15215

Female sex worker experiences with intimate partner violence screening by health care providers

Jessica L. Zemlak ${ }^{1}$, Kamila A. Alexander ${ }^{1}$, Deborah H. Wilson ${ }^{1}$, Susan G. Sherman²

${ }^{1}$ Johns Hopkins School of Nursing, ${ }^{2}$ Johns Hopkins School of Public Health

ABSTRACT IMPACT: This work will inform the need for more trauma-informed approaches to violence screenings among marginalized populations by health care providers. OBJECTIVES/GOALS: Female sex workers (FSW) experience high rates of intimate partner violence (IPV) which may have negative reproductive health consequences. Routine IPV screening by healthcare providers (HCP) is recommended. This study examines how FSW experience IPV assessments by HCP. METHODS/STUDY POPULATION: This qualitative descriptive study is nested within EMERALD, a longitudinal cohort study of street-based FSW. EMERALD assesses a structural community-level intervention on HIV and STI risks among FSW. Participants were recruited for EMERALD using time-location sampling to identify a sampling frame of venues and times where sex work is likely to occur. Inclusion criteria for this qualitative study include: participating in EMERALD, age 18-49, and willingness to participate in one phone interview. Twenty-two semistructured individual qualitative interviews were conducted. Two coders, using thematic analysis, applied deductive codes and inductive coding to identify themes. RESULTS/ANTICIPATED RESULTS: Three themes emerged from participant interviews regarding IPV screening. Inconsistent screening practices: Despite frequent reproductive health visits among participants, many women did not recall IPV screening by a HCP. Stigma as a barrier to disclosure: Women described feeling judged by HCP regarding their frequency of visits for reproductive concerns, sex work, and substance use and did not trust disclosing violence to HCP. Transactional health encounters: During visits, HCP were focused on addressing women's immediate concerns; if the HCP did ask about IPV women felt the questions were asked part of a required protocol and not driven by a concern to deeply understand their lives. DISCUSSION/SIGNIFICANCE OF FINDINGS: FSW described inconsistent IPV screening practices by HPC. Participants expressed feeling stigmatized by HCP and that encounters with HCP did not foster trust for women to disclose IPV experiences. There is a need for HPC training in universal IPV screening focused on relationship and trust building to facilitate disclosure.

17890

\section{Cardiovascular risk factors in victims of child sexual abuse}

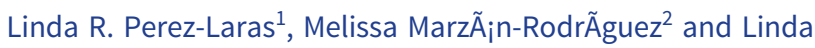
Laras $^{3}$

${ }^{1}$ University of Puerto Rico Medical Science Campus; ${ }^{2}$ Ponce Health Science University; ${ }^{3}$ San Juan Bautista School of Medicine

ABSTRACT IMPACT: The impact of this study is to encourage health professionals to screen for violent experiences as potential risk factors for CVD and adapt interventions from the non-abused in children as in adults. OBJECTIVES/GOALS: This study aims to assess the relationship between child sexual abuse and cardiovascular risk factors (CVDRF) that present in children. The objectives will provide the prevalence of CVDRF, their association with types of sexual victimization, and a score to assess the impact. METHODS/STUDY POPULATION: This study is a chart review, cross-sectional study. The Puerto Rico Health Justice Center (PRHJC) is a forensic, transdisciplinary, victim-centered, traumainformed, and evidence-based service. The demographic variables collected are age, sex; the cardiovascular risk factors variables include a level of physical activity, tobacco exposure/alcohol, vital signs (blood pressure, BMI), lipid profile, and C-reactive protein. Sexual violence variables are the type of victimization (sexual assault, sexual molestation), the number of victimizations, and the relationship with the offender. RESULTS/ANTICIPATED RESULTS: A previous study, which examined types of evidence related to the prosecution of sexual violence cases, found that among female victims, $54 \%$ was a victim of sexual assault, and $59 \%$ had at least one health concern. The study's hypothesis includes that older and female victims have a higher prevalence of cardiovascular disease risk factors. Also, children victims of sexual assault will have more cardiovascular risk factors than victims of sexual molestation. The age group, sex, number of victimizations, and relationship with the offender will also impact the relationship between the type of victimization and cardiovascular risk factors. DISCUSSION/SIGNIFICANCE OF FINDINGS: Early identification of child sexual abuse is needed to prevent long-term health impacts. The study's results will be significant in developing clinical guidelines for health care providers to identify child sexual abuse as a predictor of cardiovascular risk factors and encourage victim advocates to identify cardiovascular risk factors.

51727

\section{A Systematic Review of Implementation Science Frameworks Used in Cancer Prevention Interventions Serena Xiong ${ }^{1}$, Hamdi $\mathrm{Abdi}^{1}$ and Rebekah Pratt ${ }^{2}$ \\ ${ }^{1}$ University of Minnesota School of Public Health; ${ }^{2}$ University of Minnesota Medical School}

ABSTRACT IMPACT: Specific recommendations will be suggested in this presentation as to how a health equity lens can be applied to implementation science frameworks. OBJECTIVES/GOALS: This systematic review consolidated literature on how implementation science (IS) frameworks (e.g., RE-AIM) have been used in cancer prevention services (e.g., screening, tobacco cessation programs) 